\title{
Una mirada al discurso de la inteligencia artificial y el derecho de autor desde la teoría de la personalidad
}

\author{
$* * * *$ \\ Pablo Miguel Páez Chaljub \\ Universidad de La Sabana \\ pablo.paez011@gmail.com
}

Recibido: 30 de octubre de 2021

Aceptado: 19 de noviembre de 2021

\section{Resumen}

La inteligencia artificial es un fenómeno que ha evolucionado y se ha hecho cada vez más notable en nuestra sociedad, especialmente desde lo que se ha denominado como la "cuarta revolución industrial", un periodo caracterizado por el gran aumento en la creación de invenciones tecnológicas. Debido a este desarrollo exponencial, la inteligencia artificial ha tomado un papel especialmente relevante en nuestro día a día. Una de las formas en las que estas tecnologías se han hecho presentes en nuestra sociedad ha sido mediante la creación de obras literarias, artísticas y científicas, lo cual ha significado que vuelvan a debatirse figuras clásicas que se creían ya cerradas dentro del derecho de autor; entre estas figuras se destaca la de la autoría, la originalidad y, consecuentemente, la titularidad de derechos. El interrogante sobre esto último ha tenido diferentes respuestas que es común encontrar en distintos textos académicos, pero el objetivo de este artículo es presentar una propuesta desde los fundamentos filosóficos del derecho de autor a partir del estudio de la teoría de la personalidad, definiéndola como una de las principales teorías que pueden justificar la existencia de sistemas normativos del derecho de autor. Lo anterior, con el fin de determinar si las obras producidas por este tipo de tecnología pueden ser susceptibles de reconocimiento de derechos dentro de nuestro sistema jurídico. 
Palabras clave: inteligencia artificial (IA), derecho de autor, originalidad, personalidad, arte, apropiación, representación, cultura.

\title{
A Glance to the Speech of the Artificial Intelligence and the Author's Rights, Analyzed from the Theory of the Personality
}

\begin{abstract}
Artificial intelligence is a phenomenon that has evolved becoming increasingly notable in our society, especially since what has been called the "fourth industrial revolution", a period in which there has been a great increase of technological inventions. Due to this exponential development, artificial intelligence has taken a relevant role in daily life. One of the ways in which these technologies have become present in our society has been through literary, artistic and scientific creations, which has reopened debates, that had already been closed within the autor's rights law, over classical figures on this matter. Among these figures, the authorship, originality and, consequently, the ownership of rights stands out.

The answer about the latter has different approaches, which are commonly found in many academic texts, but the purpose of this article is to present a proposal from the philosophical foundations of the author's rights perspective, especially from the study of the theory of personality, defining it as one of the main theories that justify the existence of normative systems of autor's rights, in order to determine if the creations produced by this type of technology can be susceptible for a special recognition of rights within our legal system.
\end{abstract}

Key words: artificial intelligence (AI), autor's rights, originality, personality, art, appropriation, representation, culture.

\section{Um olhar para o discurso de inteligência artificial e direitos autorais da teoria da personalidade}

\footnotetext{
Resumo

A inteligência artificial é um fenômeno que tem evoluído e se tornado cada vez mais notável em nossa sociedade, principalmente a partir do que se chamou de "quarta revolução industrial”, período em que houve um grande aumento na criação de invençóes tecnológicas. Devido a esse desenvolvimento exponencial, a inteligência artificial tem assumido um papel relevante no nosso dia a dia. Uma das formas pelas quais essas tecnologias se tornaram presentes em nossa sociedade tem sido por meio da criação de obras literárias, artísticas e científicas, o que faz com que voltem a ser debatidas figuras clássicas que se acreditava já encerradas
} 
no direito autoral. nessas figuras, destaca-se a da autoria, da originalidade e, conseqüentemente, da titularidade dos direitos.

A resposta a esta última teve diferentes soluções clássicas, o que é comum encontrar em diferentes textos acadêmicos, mas o objetivo deste artigo é apresentar uma proposta a partir dos fundamentos filosóficos do copyright a partir do estudo da teoria da personalidade, definindo-a como uma das principais teorias que podem justificar a existência de sistemas normativos de copyright, a fim de determinar se as obras produzidas por esse tipo de tecnologia podem ser suscetíveis ao reconhecimento de direitos em nosso ordenamento jurídico.

Palavras-chave: inteligência artificial (IA), direitos autorais, originalidade, personalidade, arte, apropriação, representação, cultura.

La Inteligencia genuina debe, cuando menos, lidiar con el ruido y la furia del mundo.

(Espejo, 2020)

\section{Introducción}

La inteligencia artificial (en adelante, IA) ha pasado a integrar nuestro día a día sin darnos cuenta e incluso sin conocer con certeza de qué se trata. La publicidad y el contenido que vemos en nuestras redes sociales son la respuesta de complejos sistemas diseñados para operar de forma automática y tomar decisiones con base en los datos que suministramos, al igual que sucede con la sugerencia de palabras en los chats o el enfoque automático de la cámara de celulares y tabletas. Sin embargo, cuando preguntamos ¿qué es la inteligencia artificial? las respuestas suelen contemplar opciones que van desde un software hasta complejas y muy elaboradas máquinas humanoides, a Terminator y a David (el personaje principal de la aclamada película de Hollywood A.I. Inteligencia Artificial). Sin embargo, si algo tienen en común estas alternativas es que, en cualquier caso, una definición precisa parece distante.

La falta de conceptos claros con los que pueda existir un compromiso ha creado un marco de incertidumbre frente a las decisiones que se deben tomar para abordar una realidad que ya está desplegando sus efectos. La IA nos muestra sus virtudes en diversas áreas, 
entre las que se encuentran la medicina, la psicología y las artes. Sin embargo, hasta hace muy poco se empezaron a discutir con rigor los retos éticos, filosóficos y jurídicos que también incluye este tipo de tecnología.

La seguridad de los datos personales, la divulgación de noticias falsas y la inclusión de sesgos de distintos tipos relacionados con sexo, raza, religión, política, etc. son algunos de los puntos controversiales que han dado lugar a la necesidad de contar con sistemas de IA humanista, en los que se incluya el sentido ético que de antaño se ha predicado como necesario en la ciencia. Sin embargo, a pesar de los esfuerzos que se han hecho hasta el momento, el recorrido en búsqueda de respuestas es tan amplio como los usos que se puedan encontrar para la IA y que se hacen más numerosos día a día.

Es usual encontrar en los textos académicos distintos argumentos que no son desarrollados en profundidad por la naturaleza de las preguntas que suscitan cuando se refieren a este tipo de sistemas artificiales. El reconocimiento de derechos sobre la posible incursión en el sector de las industrias creativas no ha sido la excepción. A partir de los sistemas de IA, ha surgido un nuevo tipo de obras literarias, artísticas y científicas que constituyen uno de los temas que ha sufrido por la interdisciplinariedad y que requiere el estudio de este fenómeno; allí radican la importancia y el valor del presente estudio en el que la filosofía y el derecho se hacen uno en búsqueda de respuestas.

Cuando se estudian textos jurídicos, las preguntas filosóficas no son objeto de profundos estudios por exceder el marco de acción del derecho, y si se trata de un texto filosófico, los detalles técnicos de la actividad jurídica hacen difícil un compromiso con la realidad fáctica. Por ello, la estructura de este estudio pretende explorar con algún grado de profundidad la inteligencia artificial desde la perspectiva de la filosofía del derecho de autor. Esto, con el fin de responder a las preguntas tradicionales por la titularidad de derechos desde una perspectiva que no dependa del dinamismo del derecho, sino que dé un paso atrás para responder desde sus fundamentos.

Dicho lo anterior, el plan a seguir en el presente texto consta de cuatro puntos fundamentales. En primer lugar, se hará un breve estudio del fenómeno que vamos a estudiar, la IA, en el que el punto de partida será la definición misma de este tipo de tecnología hasta 
llegar a la forma en la que estos sistemas operan y producen obras que podrían llegar a ser protegidas por el derecho de autor.

Es así como, luego de la aproximación al fenómeno y teniendo en cuenta que la filosofía del derecho de autor no puede ser entendida de manera independiente a su historia, se dará paso al estudio de las herramientas con las que analizaremos el reconocimiento de derechos sobre las obras que surgen como resultado de un sistema de IA. Por ende, se expondrán brevemente las cuatro teorías a partir de las cuales el profesor William Fisher, de la Universidad de Harvard, afirma que puede ser justificada la existencia de sistemas protectores del derecho de autor (Fisher, 2001); la teoría de la labor, la teoría utilitarista, la teoría de la personalidad y la teoría cultural.

De las cuatro teorías mencionadas anteriormente, es posible hallar varias referencias en las que se afirman que la teoría de la personalidad es la más cercana a los sistemas de derecho de autor de los países de tradición civil por la fundamentación y defensa de los derechos morales que puede implicar. Es por ello que el tercer punto se centrará en explicar más a fondo la referida teoría de la personalidad con el fin de lograr definir la protección o no protección de obras creadas por la IA. Para esto, se desarrollarán superficialmente los argumentos de la versión clásica de esta teoría a partir de los planteamientos de Kant y Hegel, así como la versión contemporánea expuesta principalmente por la profesora Margaret Radin, de la Universidad de Michigan.

Finalmente, y con base en lo anterior, se hará un breve resumen de los argumentos principales expuestos en este escrito, para así poder ofrecer una posible propuesta o al menos abrir las puertas a la discusión sobre el reconocimiento más justo de los derechos sobre las obras creadas por IA.

\section{La inteligencia artificial y la producción de obras}

Como se mencionó, el primer interrogante a solucionar gira en torno a la necesidad de dar una definición de lo que es la inteligencia artificial, un concepto que ha sido objeto de numerosos debates desde sus primeros desarrollos. Se ha evidenciado claramente una falta de unanimidad respecto a la definición de la IA por parte de 
la academia y la doctrina, sin embargo, al estudiar los argumentos desarrollados en la literatura de este tema, es posible encontrar dos posturas respecto a lo que puede considerarse o no como un sistema de IA, a partir de lo que se ha denominado como un "sentido fuerte" o un "sentido débil" (Bringsjord y Govindarajulu, 2018).

En primer lugar, al hablar desde la perspectiva del "sentido fuerte" se hace referencia a que la IA solo puede considerarse como tal si se trata de un sistema con la capacidad de emular, replicar o incluso superar todos los procesos mentales que se desarrollan en la mente humana y/o animal. Es por lo anterior que cuando se habla de IA adoptando esta postura, es posible pensar que se trata de una "súper inteligencia” a la cual, desde un aspecto técnico, aún no hemos llegado, pero que podría considerarse como el objetivo final del desarrollo de estas tecnologías.

A diferencia de la postura anterior, desde el "sentido débil" solo podemos considerar como IA a los sistemas artificiales capaces de realizar algunos de los procesos mentales animales y/o humanos, entendiendo que la IA se limita al desarrollo de determinadas actividades con base en un sistema de estímulos y respuestas programadas.

Si bien la IA es un sistema capaz de recopilar, analizar y procesar información, independientemente de la postura en la que nos enfoquemos, se puede afirmar que el desarrollo de estas actividades trae consigo ciertas limitaciones o fallos de una u otra índole al momento de relacionarlas con el mundo exterior. Para ilustrar lo anterior, podemos pensar en un sistema de IA capaz de crear una obra de arte; si bien se puede desarrollar una tecnología que genere esta actividad de manera precisa, dicho sistema encontraría su límite en cuanto a su imposibilidad de expresar las emociones que buscaba generar en el observador al elaborar esa obra.

Ahora bien, es común pensar en el término de IA como una ciencia que genera sistemas capaces de actuar de forma independiente al ser humano, sin embargo, para Turing, el "padre de la computación moderna”, al crear el sistema binario tuvo como objetivo la capacidad resolver problemas mediante algoritmos, teniendo como base la necesidad de relacionarse de forma natural con la intervención del ser humano.

Conforme al paso del tiempo, se ha logrado un gran aumento 
de capacidad de procesamiento en este tipo de tecnologías, esto es, la creación de sistemas de IA con la capacidad de procesar grandes bases de información en un tiempo considerablemente inferior al que le tomaría a un ser humano el realizar esta acción. A raíz de este progreso, surgieron varias características esenciales con el fin de seguir aumentando la eficacia y eficiencia de estas tecnologías. Las características mencionadas se pueden observar en la siguiente imagen:

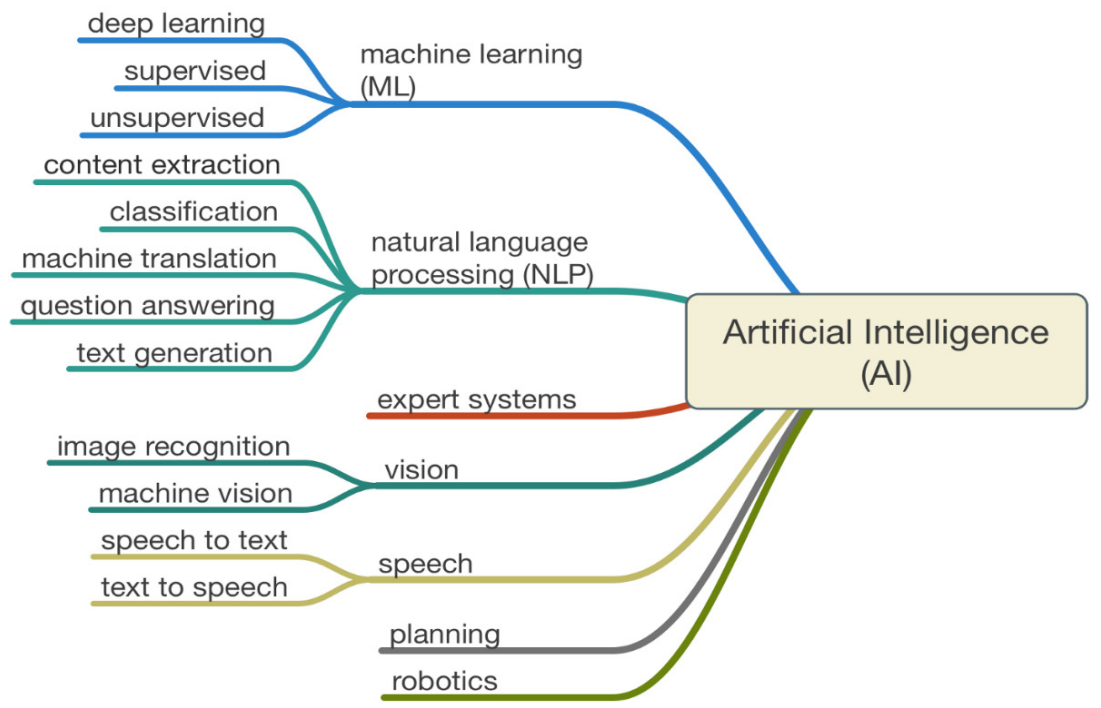

Figura 1. Características de la IA (fuente: Mills, 2016).

Ahora bien, dicho aumento de capacidad de procesamiento no ha sido la única característica que fue objeto de progreso, junto con ella encontramos el mejoramiento de la capacidad de extraer información mediante análisis estadísticos y la capacidad que han desarrollado estas tecnologías para la toma de decisiones por medio del planteamiento de predicciones, las cuales pueden ser perfeccionadas dentro de las redes neuronales profundas a través del entrenamiento automatizado, también conocido como deep learning.

Es así como la creación de sistemas de IA cada vez más complejos y especializados ha alcanzado procesos de entrenamiento autónomo, en otras palabras, el aumento de la capacidad de procesamiento ha permitido que los sistemas artificiales sean capaces de procesar, 
predecir y decidir de forma autónoma, así como de aprender de la experiencia de este tipo de actividades en búsqueda de un funcionamiento cada vez más óptimo.

Así, al analizar las decisiones de la IA desde un punto de vista técnico, muchas veces cuesta, incluso para los ojos más expertos, entender de dónde provienen los resultados del procesamiento de información. Cuando estos sistemas tan complejos vinculan de forma automática unos datos con otros, trazando rutas que, en ocasiones, podrían incluso no haber sido consideradas por el diseñador del sistema de IA, se crea un marco en el que la trazabilidad de cada uno de los elementos que dieron lugar a que el sistema tomara una decisión se vuelve imposible. A esto se lo ha denominado black box theory. En otras palabras, debido a la extrema complejidad de los procesos llevados a cabo en las redes neuronales profundas del sistema y a su capacidad de entrenamiento autónomo, muchas veces resulta imposible para el ser humano acceder a la totalidad de la cadena causal detrás de las decisiones que toma un sistema de IA y esto, como es de esperarse, genera un elemento de impredecibilidad sobre las creaciones de estas tecnologías; ¿supone esto que debamos hablar en términos de originalidad cuando el resultado del procesamiento de información es una obra artística?

Es importante recalcar que la definición de la IA, junto con sus características, puede ser objeto de un análisis mucho más extenso debido a la gran complejidad de estos sistemas y a todo el desarrollo que se ha generado en torno a estas tecnologías. Sin embargo, para los fines de este artículo, el propósito no es otro que el de construir una imagen mental de la IA que se desprenda de las ideas cliché sobre un sistema robótico humanoide. La IA es mucho más que eso, es procesamiento de información a gran escala con unas complejidades técnicas que hoy ya sobrepasan nuestra capacidad de comprensión cuando buscamos responder a los porqués que pueden surgir frente a los resultados que nos arrojan estos sistemas.

The Next Rembrandt, el ejemplo más citado cuando hablamos de la creación de obras artísticas por sistemas de IA, es una muestra de lo lejos que han llegado este tipo de desarrollos tecnológicos y de la brecha de conocimiento que parece crecer cada vez más cuando buscamos entender el porqué de las decisiones que hemos ido dejando 
a merced de la IA. ING y Microsoft, con colaboración de la Universidad Técnica de Delft, del Museo Mauritshuis de La Haya y del Museo Casa de Rembrandt de Ámsterdam, se unieron para diseñar un sistema capaz de crear, si es que este es el término correcto, un nuevo cuadro de Rembrandt. El resultado, casi 350 años después de la muerte del pintor neerlandés, es un nuevo cuadro digno del estilo, técnica y capacidad artística de uno de los artistas más renombrados en la historia del arte.

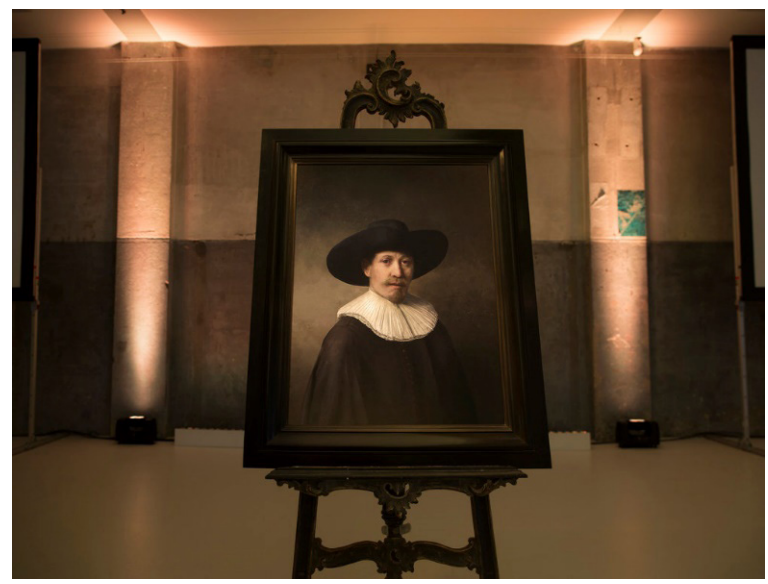

Figura 2. The Next Rembrant, creado en colaboración por ING, Microsoft, la Universidad Técnica de Delft, el Museo Mauritshuis de La Haya y el Museo Casa de Rembrandt de Ámsterdam (fuente: The Next Rembrandt). ${ }^{1}$

Es de aquí de donde se desprende nuestro interrogante principal: ¿cuál es la forma más justa de reconocer derechos sobre este tipo de creaciones? Para responder, o al menos para intentar acercarnos a una respuesta, veamos los elementos a partir de los cuales debería fundamentarse cualquier tipo de respuesta con la que podamos generar algún tipo de compromiso.

\section{El fundamento filosófico del derecho de autor}

Como habíamos anunciado al inicio del texto, la segunda parte del

1 Disponible en: www.nextrembrandt.com. 
escrito tiene el propósito de profundizar en la justificación filosófica para dar fundamento a la necesidad de contar con sistemas protectores del derecho de autor. Para cumplir con este fin, se explicará cada una de las cuatro teorías recopiladas por el profesor William Fisher, de Harvard, en su artículo "Theories of Intellectual Property" (Fisher, 2001): la teoría de la labor o del trabajo, la teoría utilitarista, la teoría de la personalidad y la teoría cultural.

Llegados a este punto, es preciso aclarar que este apartado en ocasiones puede resultar difuso. Sin embargo, es importante que recordemos que su lectura es necesaria para comprender de una mejor manera el alcance y las fronteras de la teoría de la personalidad para el posterior estudio de la creación de obras generadas por IA desde sus planteamientos principales.

\subsection{Teoría de la labor o del trabajo}

Esta teoría ha tenido un gran desarrollo a lo largo de la historia, encontrando sus argumentos iniciales en la obra de Locke, uno de los primeros autores en aplicar los principios del derecho natural al reconocimiento de la propiedad privada. Esta teoría puede resumirse en que la titularidad de la propiedad privada recae sobre la persona que realizó el esfuerzo o el trabajo en cuestión para transformar elementos de la naturaleza. Según Fisher (2001), la justificación de la teoría de que un objeto puede pasar de ser considerado como propiedad común a pertenecerle a un individuo, de acuerdo con Locke, encuentra sus bases teóricas en los seis puntos, los cuales se han resumido de la siguiente manera (Páez, 2021):

i. La existencia de la "razón natural", según la cual el hombre tiene el derecho a buscar su preservación y esto solo puede lograrlo mediante la individualización y apropiación de distintos elementos que le permiten hacerse con comida y abrigo.

ii. El factor religioso dentro de esta teoría propone, según Fisher (2001), que existe una concordancia entre la existencia de la "razón natural” y la condición de vivir dentro del mundo. Esto, debido a que si bien Dios creó el mundo para que los seres humanos viviéramos en él en comunidad, también lo hizo mediante una instrucción de dominarlo para mejorar la vida, algo que solo 
puede lograrse mediante la inversión del trabajo y la apropiación de los frutos que surgen de este.

iii. as intuiciones sobre el ser dueño de sí mismo apunta a que los frutos del trabajo también deben entenderse como una cierta "extensión" de esta propiedad. Si cada quien "es dueño de su cuerpo y del trabajo de sus manos", consecuentemente todo aquello que mezcle con ese trabajo, debería ser suyo también.

iv. Dios les dio el mundo a los trabajadores y a los racionales, no a la fantasía y la codicia de los contenciosos. Por esta razón, se entiende como un complemento al punto anterior que quien invierte su trabajo debe ser reconocido como dueño.

v. Un sentido de proporcionalidad y de justicia que surge al entender que el valor de las cosas que son útiles a los hombres está determinado principalmente por el trabajo que se ha invertido en ellas y no por el valor de la materia de la cual están hechas.

vi. El criterio de transformación productiva que se deriva del trabajo. Cuando hay un esfuerzo que se aplica a una materia prima, no puede hablarse únicamente de la existencia del producto como consecuencia de la existencia de los materiales que lo componen en el mundo, sino como el fruto de un proceso de transformación gracias al cual se da un orden, o se dota de un propósito a lo que en principio era caótico e inútil.

Analizando lo anterior, Fisher (2001) concluye que si bien puede justificarse la propiedad sobre un objeto material, esta línea argumentativa se queda corta al intentar aplicarla a creaciones del intelecto. Pues al intentar identificar los argumentos principales de los puntos anteriores se pueden tomar dos caminos: el primero sería entender como primordiales los argumentos i y ii expuestos anteriormente, lo cual genera un sustento débil respecto a la propiedad intelectual. A diferencia de esto, si se escogen como esenciales los puntos iv y v, sí podría hablarse de una correlación entre el trabajo y las creaciones materiales o inmateriales.

El problema anterior deriva de que Locke (1998) desarrolló su teoría basándose en el concepto general de "propiedad", mas no en la propiedad intelectual específicamente, lo cual es importante tener en mente para poder entender por qué han surgido diversas críticas 
hacia esta teoría, como por ejemplo el problema lógico que plantea Moore (2015), evidenciando la dificultad de correlacionar una acción proveniente del ser humano frente a un objeto ajeno a este.

Por otro lado, Fisher (2001) va más allá y plantea otro reto a esta teoría: el interrogante sobre qué se entiende por "trabajo intelectual", respondiéndose a sí mismo con cuatro posibles respuestas, pero, a su vez, planteando los problemas de estas de la siguiente forma:

1. Se entiende como la relación entre el esfuerzo y el tiempo invertidos en la creación: esta respuesta es la más parecida a la teoría de Locke (1998), pero debe tenerse en cuenta que no fue desarrollada para aplicarse a la propiedad intelectual, sino a la propiedad en general.

2. Es aquel que hace referencia a una actividad con la cual el productor no está comprometido: sostener este punto es problemático ya que no se podría proteger a aquellas personas que se dedican por completo a estas actividades.

3. Se refiere a la actividad que tenga por objeto alcanzar un beneficio social (Hugues, 1998): este punto sostiene que es necesario el elemento de utilidad de las creaciones, lo cual también limitaría en gran forma la protección que puede brindarse.

4. Que sea una actividad creativa: se hace referencia a que la creación no sea obvia, lo cual también es debatible frente a las obras artísticas.

Resumiendo lo anterior, las críticas tienen un punto en común y es el debate sobre la limitación de la protección que se generaría al adoptar esta teoría, pues al asumir el reconocimiento de un derecho natural y exclusivo sobre las creaciones, conlleva la limitación de acceso a estos por parte de terceros y, en el caso específico de la propiedad intelectual, ocasionaría el aumento de monopolios, restringiendo el acceso a determinadas creaciones que, en principio, deberían ser de uso o conocimiento común.

Finalmente, aunque resulta claro que la teoría de la labor cuenta con varios puntos, matices y oportunidades para el debate respecto a su aplicación sobre la propiedad intelectual, es importante resaltar la importancia de su existencia, pues sentó las bases de las demás teorías que irían apareciendo con el paso del tiempo. 


\subsection{Teoría utilitarista}

Esta teoría, en resumidas cuentas, busca como elemento esencial la obtención de beneficios y riqueza para la comunidad a través de cualquier tipo de conducta en la que se pueda incurrir, o cualquier medida que se pueda tomar para regular las relaciones entre los miembros de una comunidad. En otras palabras, que las personas encargadas de redactar normas y/o políticas públicas lo hagan con base en la máxima de "el mayor bien para el mayor número" y los planteamientos principales en las obras de Jeremy Bentham y John Stuart Mill.

Fisher (2001), para explicar esta teoría, expone como elemento esencial un criterio similar al llamado kaldor-hicks, según el cual, para escoger entre un sistema y otro, se debe evaluar y señalar como la mejor opción aquel que, al mover el estado de cosas en cuestión, otorgue mayores beneficios y compense las pérdidas que puedan ocasionarse por dicho cambio de sistemas.

Si bien esta teoría se preocupa fuertemente por la obtención de un beneficio monetario, no se reduce solo a esto. Es correcto afirmar que, en el ámbito de la propiedad intelectual, se han desarrollado varios argumentos que conllevan o no como criterio relevante la retribución material, siendo algunos de estos los siguientes:

Perspectiva de la recompensa o reward theory: esta perspectiva se centra en la recompensa obtenida por la inversión de recursos y esfuerzos durante el proceso creativo en beneficio de la comunidad. Esta premisa podría ser comprendida con mayor facilidad si consideramos el límite de tiempo que se le otorga a la protección de los derechos patrimoniales del autor, ya que los derechos de exclusividad encuentran su razón en la justa recompensa por los esfuerzos invertidos para crear la obra, pero que, en último término, pretenden que el conocimiento allí contenido se convierta en un canal de transmisión de dominio público.

Este mismo fin se encuentra en los sistemas de patentes y diseños industriales, por ejemplo, en virtud de que existe un agente que lleva a cabo un proceso creativo a cuyo término obtiene como resultado un producto sobre el cual el sistema normativo le reconoce unas facultades y prerrogativas exclusivas, siempre y cuando sea a cambio de ofrecer a la sociedad el avance que representa su producto, de allí que esta serie de derechos sean temporales; y su registro, no renovable. 
Lo anterior conlleva un flujo de información en la sociedad, entendiendo que la comunidad, en un primer momento, actúa como un sujeto pasivo frente al producto en cuestión, pero una que vez dicha creación entre al conocimiento común, puede llegar a actuar como sujeto activo al producir nuevos productos con base en este conocimiento adquirido de la primera. Es por ello que desde esta teoría puede entenderse por qué la propiedad intelectual puede ser considerada como un medio de desarrollo.

Por otro lado, es importante señalar que dentro de esta perspectiva se encuentran varias posturas intermedias, como la llamada desert-labour theory, la cual integra la teoría de la labor y el reconocimiento de una recompensa que surge a raíz del esfuerzo implementado por el autor para la creación de determinado producto.

Perspectiva del incentivo: esta teoría tiene como objetivo principal promover la creación de productos a partir de ciertos privilegios otorgados a quienes desarrollan dichas creaciones; esto, con el fin de aumentar la actividad de creación e innovación, lo cual repercute directamente en la sociedad aumentando el conocimiento científico, tecnológico y cultural, dándole una importancia primordial al desarrollo y avance de la propiedad intelectual.

Es por lo anterior que puede considerarse la existencia de dos tipos de incentivos: en primer lugar, se le otorga un reconocimiento de derechos en cabeza del autor, lo cual genera una propuesta económica para sobrellevar o para equilibrar el alto costo de producción y el bajo costo de distribución de esa creación. En otras palabras, el modelo de incentivos ayuda en gran manera a compensar dichos costos. En segundo lugar, se encuentra un incentivo que tiene como fin conocer el mercado en el que se está actuando, esto es para que los desarrolladores puedan crear nuevos productores conforme a las expectativas de los futuros usuarios, asegurando lo que Fisher (2001) llama la "optimización de patrones de productividad".

Perspectiva de las invenciones rivales: dentro de la teoría utilitarista se encuentra una vertiente que tiene como elemento esencial la competencia entre rivales, con el fin de fomentar el progreso innovador en la sociedad, según lo explican Fisher (2001) y Menell (2000).

Esto se materializa en la rama empresarial en tres etapas. La primera surge en el momento en el que un productor genera una in- 
vención susceptible de protección por el sistema de propiedad intelectual, logrando así una clase de "monopolio" temporal sobre la explotación exclusiva de la creación; con esto se da paso a la segunda etapa, en la que se inicia una competencia entre otros productores con el fin de alcanzar una ventaja mediante la creación de otra obra que supere a la creada por el primer productor; $y$, finalmente, dichos productores utilizan sus nuevas creaciones, susceptibles de protección, para obtener ganancias en el mercado.

Teniendo en cuenta la definición de la teoría utilitarista y las perspectivas que se encuentran dentro de esta, se da paso a exponer brevemente ciertas críticas que ha recibido esta teoría. La primera a la que se hará referencia es aquella que argumenta la incertidumbre que hay en torno a los medios adecuados para lograr el objetivo de esta teoría, lo cual presenta un gran reto junto al ideal de aplicar medidas proteccionistas con el fin de aumentar o de incentivar la producción nacional. La segunda crítica se posa sobre la posible limitación del acceso a los productos creados dentro del mercado; dicha limitación surge debido a la concesión de derechos para la explotación exclusiva. Se critica que, en caso de que el aumento de obstáculos impuestos por el sistema de propiedad intelectual sea mayor que el objetivo de promover dicha actividad inventiva, se afectaría negativamente a la sociedad al impedir "el mayor bien para un mayor número", pues no se lograría una producción que alcance estas expectativas.

\subsection{Teoría de la personalidad}

Esta teoría, en su desarrollo desde un punto de vista clásico, se deriva principalmente de Kant (1887) y Hegel (1991) y, desde su desarrollo contemporáneo, por otros autores, dentro de los cuales se resalta a la profesora Margaret Radin (1982). Si bien antes de explicar esta teoría se debe tener en cuenta que existen diversos enfoques que la definen, los cuales serán explicados más adelante, se rescatan dos elementos que priman sobre los otros: el objetivo de lograr un desarrollo de la naturaleza humana y la importancia de la personalidad y de la autonomía.

En primer lugar, la búsqueda de alcanzar un desarrollo mayor de la naturaleza humana encuentra su fundamento en la afirmación de 
que el ser humano siempre ha buscado dominar su entorno, adaptarlo a sus necesidades. Dicha dominación surge de la necesidad de asegurar su supervivencia satisfaciendo sus necesidades, buscando así una vida más digna (Dworkin, 1978) y, como postula Menell (2000), en ser propietarios de los objetos para alcanzar este fin.

Se debe poner en conocimiento que, a diferencia de los autores anteriores, existen otros que no fijan su atención en la propiedad como protección únicamente de la dignidad humana; según expone Drahos (2016), el concepto de "propiedad" también puede ser entendido como un medio para la soberanía. Estos planteos son recogidos por Radin (1982), que los incluye en su teoría contemporánea como recursos externos, afirmando que, para ser persona, es necesario tener el control de estos elementos externos del entorno en el que se habita.

Teniendo presente este primer elemento en común, es preciso destacar la importancia de la personalidad y de la autonomía dentro de esta teoría. De los planteamientos de Radin, Du Bois (2018) afirma que existen formas de propiedad con mayor valor y otras con un valor menor, lo cual se distingue entre la propiedad material y la propiedad inherente a la personalidad del propietario.

Radin (1982) argumenta que la propiedad intelectual debe asegurar una mayor protección, pues esta se deriva de la cercanía de la creación con la personalidad de quien la creó. Para ilustrar lo anterior, esta autora utiliza dos ejemplos. El primero hace alusión a un anillo de compromiso, el cual sería un bien fungible, ${ }^{2}$ pero encuentra un valor mayor por el vínculo y el significado que este genera en la persona que lo usa. El segundo ejemplo se refiere al valor de una casa, la cual, según Radin (1982), debe contar con una mayor protección por estar ligada a la privacidad y a la libertad.

Ahora bien, Hughes (1998) considera que "la propiedad provee un mecanismo único o especialmente adecuado para la autorrealización,

2 Aquellos objetos comerciables que "suelen determinarse según su número, medida o peso, y que, por regla general, son sustituibles; esto es, se toman en cuenta solo su medida y calidad, pero no individualmente como el dinero, los granos, el vino, los libros en rústica, y con frecuencia, aunque no siempre, los títulos de valor" (Carrión, 1982, como se citó en Velázquez, 2004). 
la expresión personal, y para la dignidad y el reconocimiento como una persona individual". Respecto a la propiedad intelectual, centrándonos en el derecho de autor, este postulado solo puede aplicarse al afirmar que la propiedad tiene un carácter personal, adquiriendo un mayor valor al ser relacionada con la personalidad del creador.

Desarrollando lo anterior y tomando el postulado de Locke (1988) de que la propiedad está al servicio de la comunidad, TreigerBar-Am (2008) expone que el reconocimiento de la propiedad sobre determinada persona puede derivarse de la huella que esta le deja, la cual no solo contiene el elemento de la personalidad, sino que también incluye los elementos de individualización y originalidad que emergen de la autonomía del creador. Este autor, para sustentar lo anterior, se refiere a la teoría de la personalidad de Kant, ${ }^{3}$ la cual, desde su análisis, también encontraba su sustento en la individualidad y originalidad de la creación protegida.

Lo anterior es un resumen de los puntos principales de esta teoría, pero vale la pena recordar que se explicará de forma detallada más adelante. En el apartado dedicado específicamente a ella se adentrará en los planteamientos de Kant y de Hegel como exponentes principales de la noción clásica y, a su vez, se expondrá en detalle la noción contemporánea desde la teoría de Margaret Radin como su máxima exponente.

\subsection{Teoría cultural}

Esta teoría, si bien es la menos conocida, es con la que Fisher (2001) encuentra una mayor afinidad. Para definirla, este autor expone los tres argumentos principales que tiene en común esta teoría con las explicadas anteriormente. El primer punto se refiere a la importancia que se le da al bienestar de la sociedad (teoría utilitarista). La teoría cultural retoma esto añadiéndole la necesidad de la creación, por parte de los individuos, de todo un sistema cultural, en cuanto a que el beneficio no puede ser buscado de manera individual, sino que debe ser alcanzado como una colectividad. En segundo lugar, se encuentra la importancia que esta teoría le da a la satisfacción de

3 Que será explicada en mayor detalle el siguiente apartado. 
las necesidades humanas (teoría de la personalidad), con el fin de fomentar la actividad creativa en la sociedad construyendo así un sistema integral.

Lo anterior, teniendo en cuenta que estos desarrollos inventivos responden al entorno del autor de dichas invenciones, pues se entiende que este es un individuo perteneciente a una sociedad y que su proceso creativo es influenciado en mayor o menor medida por la cultura que lo rodea. En consecuencia, el deber de las instituciones es el de garantizar el entorno adecuado para que las personas puedan tener una vida buena (Fisher, 2001) y que exista una justicia distributiva con el fin de influir en estos procesos creativos individuales que terminan haciendo parte de la construcción de un bien mayor en la sociedad.

Según Fisher (2001), el elemento de la vida buena puede ser definido dentro de los siguientes componentes básicos: vida, salud, autonomía, compromiso, expresión personal, competencia, conexión y privacidad. Por otro lado, la justicia distributiva se entiende como la capacidad de acceder a aquellas condiciones necesarias para el desarrollo pleno de cada persona.

El problema de esta teoría surge al momento de intentar aplicar los elementos de la naturaleza humana, la vida buena y la justicia distributiva a la realidad de la sociedad; surge la incógnita de cómo materializar de forma adecuada y práctica dichos conceptos con el fin de que no se queden solo como un "deber ser". Para dar respuesta a lo anterior, la teoría de la cultura integra un cuarto elemento en la fórmula: la correlación entre la educación, el arte, la diversidad y la democracia ${ }^{4}$ (Fisher, 2001) en una sociedad, llamándose "cultura”.

Este elemento se entiende dentro del contexto en el que los autores producen determinada invención, si bien en primera instancia se puede afirmar que el proceso creativo es individual, no se puede negar que este ha sido influido por el entorno en el que está inmerso,

4 Sobre esto, Fisher (2001) explica que al hablar de la democracia no se hace solo bajo el entendido de la participación en la vida política, sino de una democracia en sentido semiótico. En otras palabras, hace referencia a un sistema en el que todos los individuos pueden ser partícipes en la construcción de una tradición cultural de forma activa. 
tomando como beneficio e inspiración otras invenciones anteriormente creadas. La influencia de este conjunto de bienes y valores se denomina como el "acervo común", en otras palabras, se puede afirmar que el autor, en su proceso inventivo, es influenciado y utiliza en su beneficio el acervo común y, en consecuencia, dicha innovación pasa a construir y a ser parte del acervo común de esa sociedad.

Concluyendo lo anterior, se puede afirmar que el derecho de autor cuenta con un respaldo filosófico que ha atendido a distintas necesidades que han ido apareciendo con el paso del tiempo. En atención a esto, existen al menos las cuatro teorías que han buscado responder esas cuestiones fundamentales para el desarrollo y avance de los sistemas de protección de propiedad intelectual y derecho de autor. Muchas veces olvidamos que el derecho es dinámico, pero este dinamismo debe estar justificado desde unos principios filosóficos que aseguren el encuentro de las soluciones más justas.

\section{La teoría de la personalidad}

Ahora bien, la tercera parte de este escrito se centrará en el estudio detallado de la teoría de la personalidad, entendiéndola como la teoría que más se acerca a los sistemas de tradición civil. Se expondrá desde sus tres puntos de vista: dos desde la visión clásica, desde los postulados de Kant y Hegel, y el ultimo desde la visión contemporánea, deteniéndonos especialmente en esta perspectiva desarrollada principalmente por la profesora Margaret Radin. Lo anterior, con el fin de poder concluir en el apartado final del texto de qué manera esta teoría puede dar luz sobre el reconocimiento de derechos en la protección de las obras creadas a través de la IA.

\subsection{Teoría kantiana del derecho de autor}

Antes de iniciar con la exposición de esta perspectiva, es preciso tener en cuenta que la teoría de Kant, aunque recaiga sobre la propiedad y el derecho de autor, no alcanza a englobar por completo lo expuesto en la teoría de la personalidad. Debido a lo anterior, el estudio a esta justificación de la existencia de sistemas protectores de derecho de autor y de propiedad intelectual debe realizarse bajo 
un análisis doctrinal integrativo entre la teoría kantiana y la versión hegeliana, con el propósito de alcanzar una definición más robusta de la concepción clásica de esta teoría.

Con respecto a la versión kantiana, se puede afirmar que encuentra su fundamento en la teoría moral de este mismo autor, especialmente por los elementos de libertad, autonomía (Treiger-Bar-Am, $2008)^{5}$ y personalidad (Stengel, 2004), incluidos por el pensador en sus obras.

Con respecto a lo anterior, en su obra titulada la Filosofía del derecho, Kant (1887) explica el vínculo entre la libertad y la propiedad, justificándolo desde una doble visión de la libertad: como acción y omisión, entendiendo esta última como la no interferencia con el ejercicio pleno del derecho de propiedad en cabeza de otros. ${ }^{6} \mathrm{La}$ libertad, en relación con la propiedad, se entiende como una acción, ya que es la capacidad de apropiarse de determinado objeto, siempre y cuando sea el primero en poseerlo; esto, debido a que el reconocimiento de la propiedad se relaciona directamente con la existencia y de la disponibilidad de dicho objeto.

Continuando con esta línea argumentativa, Kant (1887) define el concepto de "posesión" en dos facetas: en primer lugar, desde un punto de vista empírico, entendido como el vínculo entre el objeto situado en un lugar externo al sujeto, también llamada "posesión sensible"; en segundo lugar, nos encontramos con la llamada "posesión racional", la cual se refiere al proceso mental que ocurre al interior del individuo al distinguir su vínculo con dicho objeto como algo externo a él. Esta justificación tiene gran relevancia en el desarrollo de Kant en cuanto a que es la primera vez que el autor incluye un aspecto que sobrepasa lo material, dejando en evidencia la necesidad de distinguir entre la obra meramente material y el contenido de esta.

5 Treiger-Bar-Am (2008) afirma que la protección de los sistemas de copyright es una consecuencia del modelo kantiano de libertad. La libertad de expresión, por las implicaciones de su ejercicio, se relaciona con la autonomía, conforme la cual el autor afirma que es posible hablar de una influencia kantiana no solo en los sistemas de derecho de autor de tradición civil, sino también en los sistemas de copyright.

6 Stengel (2004) afirma que se trata de una regla aceptada a priori por la sociedad. 
Como ejemplo, Kant (1973) expone el caso en concreto de un libro, afirmando que el autor de este sería el que profirió determinado discurso mediante signos del habla. Lo anterior genera ciertos privilegios sobre el autor de dicha obra, el cual puede decidir si autoriza o no a un tercero, entendido como editor, para que plasme ese discurso en un bien material dirigido al público. Esta autorización es la que le otorga cierto derecho al editor, esto es, dicho derecho depende, en primer lugar, del derecho del autor para autorizar o no la edición de su discurso.

No obstante lo anterior, Treiger-Bar-Am (2008), citando a Geller (2000), llega a la conclusión de que Kant se limitó a explicar la propiedad respecto a un discurso, dejando fuera otras formas de arte que también merecen este tipo de protección frente a reproducciones no autorizadas. Frente a esto, Kant (1973) argumenta que las obras literarias son las únicas susceptibles de este tipo de protección, dándole un papel de suma importancia a la expresión personal del autor literario.

Dentro de este marco, se entiende que el derecho a la propiedad no hace referencia únicamente al corpus mechanicum, pues también se le reconoce dicho derecho a los autores respecto a la parte de ellos mismos que plasman en determinada obra. Este argumento crea diversos retos, que Kant (1973) también reconoce, pues suena contradictorio que la autorización para enajenar la parte material de la obra conlleve al mismo tiempo cierta posesión sobre una parte del autor mismo. Problemática que no llega a ser resuelta hasta la aparición de Hegel.

Continuando con la teoría kantiana, si bien es evidente que da una mayor relevancia a las obras literarias, se debe resaltar que dicho papel no se justificaba en el argumento de que la literatura fuera jerárquicamente superior a otras artes; Kant (1973) defendía esta distinción en cuando a que la literatura, a diferencia de las otras artes, no tiene como elemento esencial su representación inmediata.

Ahora bien, Kant (1973), en la Crítica del juicio, señala que el arte nace a raíz de un hacer productivo, entendiendo el arte como "la producción por medio de la libertad, es decir, mediante una voluntad que pone razón a la base de su actividad" (Kant, 1973). Lo cual puede resumirse en el planteamiento de que una obra puede ser 
considerada como artística siempre y cuando sea producto del ser humano, en cuanto a que en el autor ya existía una representación preconcebida antes del desarrollo de la obra misma (Kant, 1973).

Para evitar que lo anterior amplíe el concepto del arte de tal manera que cualquier oficio o rama de la ciencia pueda encuadrarse dentro de él, Kant justifica la distinción del arte con otras ciencias en cuanto a que: (i) la cantidad de conocimiento que se llegue a tener de este no garantiza la capacidad de producir una obra artística; y (ii) la creación artística se deriva de la libertad, a diferencia de un oficio que usualmente se realiza por necesidad, mas no por libertad (Kant, 1973).

Es importante resaltar que en su obra le da una mención especial al tema del "arte bello como arte del genio", lo cual podría ser contradictorio en el intento de explicar el derecho de autor, debido a que este busca proteger las obras independientemente de su nivel de "belleza" ${ }^{7}$ En todo caso, el análisis del término expuesto por Kant debe ser interpretado a la luz de lo postulado por Treiger-Bar-Am (2008), en el sentido de que esta categorización no responde a una belleza estética, sino a ciertos elementos que pueden encontrarse en la teoría kantiana y en el sistema de derecho de autor actual. ${ }^{8}$

Desarrollando lo anterior, en primer lugar Kant distingue el "arte mecánico" del "arte estético", refiriéndose al primero como el conocimiento adquirido del artista y la acción para materializar dicho conocimiento. Por otro lado, al incluir el elemento de un sentimiento de placer por parte del autor, se deja de lado la fase inicial mecánica para entrar al plano del denominado "arte estético". Con esto se deja claro que el concepto del "arte bello" no genera un obstáculo para aplicar esta teoría al derecho de autor, pues este elemento surge internamente en el creador de la obra, mas no se predica de la obra en sí misma.

7 Muestra de ello es que, durante la fase de internacionalización del derecho de autor, el Convenio de Berna y los distintos tratados posteriores cuentan con artículos que establecen expresamente esta salvedad.

8 Treiger-Bar-Am (2008) le da claridad a este término al establecer que no se trata de las artes bellas en sentido estético, sino de las bellas artes en relación con la forma de "obras artísticas", desconociendo que el arte se puede predicar incluso en otro tipo de esferas según la forma en la que una persona ejecuta una labor. 
Respecto al papel del "genio" (Treiger-Bar-Am, 2008), no puede afirmarse que va en contradicción del derecho de autor, en cuanto a que Kant (1973) define este término como "la capacidad espiritual innata mediante la cual la naturaleza da la regla del arte". Dicha capacidad de producción no está limitada a determinadas reglas, por lo cual está directamente relacionado con el factor de originalidad de las obras. En otras palabras, la genialidad significa originalidad, el genio no puede producir arte siguiendo un procedimiento establecido, diferenciándose de la ciencia, ejerciendo así una libertad que se plasma en la capacidad del autor de usar su conocimiento con el fin de crear una obra original a través de la imaginación y el entendimiento que ha adquirido (Kant, 1973). Lo anterior, con el fin último de que el conocimiento generado a través de las bellas artes se transmita y perdure en el tiempo (Kant, 1973).

\subsection{Teoría hegeliana}

En segundo lugar, dentro de la perspectiva clásica, se expone la teoría del derecho de autor, específicamente la de la personalidad, desarrollada por Hegel. Resulta claro, al estudiar los textos de Drahos (2016) o Hughes (1998), que la teoría de la personalidad encuentra su fundamento, en gran medida, gracias a la obra de Hegel.

Retomando las interrogantes que deja la teoría kantiana, nos enfocaremos en el caso en que el autor le otorgue a un tercero la autorización para enajenar su obra (corpus); la problemática surge debido a que dicha autorización no solo incluiría la enajenación del corpus, sino también, en cierto modo, de una parte del mismo autor (ius personalissimum), al entender la obra como una extensión de su creador, lo cual resulta inmoral y contradictorio al sistema de derecho de autor.

Para dar respuesta a lo anterior, Hegel se centró en entender cómo funcionan los sistemas de protección de estos derechos en la actualidad, apartándose de la teoría kantiana al afirmar que el valor de la propiedad no se deriva de la relación con el autor; por el contrario, dicho valor se encuentra en el concepto mismo de propiedad como regulador de las relaciones entre el propietario y los terceros (Stengel, 2004). Este enfoque permite afirmar que la teoría hegeliana tiene 
como propósito garantizar una protección mayor de la propiedad intelectual en el sentido de que el derecho reconocido va más allá de la capacidad del autor de disponer del objeto material.

Esta teoría le da gran valor a la voluntad y a la libertad, coincidiendo con Kant; sin embargo, Hegel (1991) se detiene a mencionar que la voluntad, en la medida en que es universal y autoconsciente, carece de contenido, por esto es necesario que se encuentre un objetivo determinado que guíe la voluntad, desarrollando así el yo en la personalidad. En resumen, el lograr salir de esa indeterminación y el desarrollo de la personalidad del individuo en su entorno depende de que este anteponga la voluntad sobre cualquier cosa, apropiándose de ella (Hegel, 1991), convirtiendo la propiedad en la encarnación de la personalidad (Stengel, 2004).

Conforme a esto, los objetos terminan siendo la materialización de la personalidad individual y la esencia universal de la autoconciencia (Yoo, 2019), logrando a través de ellos la existencia de la voluntad desde la libertad; por ende, la libertad es esencial para la apropiación de determinados bienes con el fin de la subsistencia de la persona (Stengel, 2004).

Teniendo en cuenta lo expuesto anteriormente, se analizará esta teoría aplicada a las creaciones intelectuales. Hegel da a conocer en su obra Lineamientos de la filosofía del derecho su interés en incluir las obras del intelecto como susceptibles de protección en cuanto al elemento de la personalidad encontrado en estas creaciones.

Hegel se detiene para preguntarse cómo es posible que una creación del intelecto pueda ser vista como objeto de un contrato, y se responde a sí mismo afirmando que el artista inicia su proceso de creación de manera interna (inmaterial y espiritual) conforme a las características del espíritu libre, el cual, a su vez, tiene la capacidad de superar dichas barreras con el fin de materializar en forma de expresión los conocimientos del autor (corporeidad y existencia material). De tal forma, Hegel elude el problema ético planteado desde la teoría de Kant, al diferenciar el objeto del espíritu de su mera materialización, lo cual permite la enajenación de estas obras en cuanto a que se entienden como un objeto externo a su autor (Hegel, 1991).

En relación con la idea anterior, tampoco se puede ir al otro extremo de argumentar una inexistencia de vínculo entre el autor y la 
obra material. Al respecto, Hegel deja claro que los autores de las obras susceptibles de enajenación siguen manteniendo ciertos derechos sobre estas. Esto significa que una vez que se haya enajenado la obra material, el autor sigue siendo propietario de su forma de expresión original y distintiva. Se distingue la compra del objeto (derecho real) del contenido inmaterial de la creación, pues el derecho real adquirido mediante la compra de un objeto no significa que el comprador también obtenga el derecho que se predica sobre la forma de expresión del contenido, sustentando la necesidad de ampliar la protección de la propiedad intelectual prohibiendo la realización de copias no autorizadas por el autor de la obra.

Siendo así las cosas, Hegel (1991) procede a desarrollar el término de las obras derivadas y del plagio. En Lineamientos de la filosofía del derecho, el autor afirma que si bien el objetivo de las creaciones es lograr que sean puestas en conocimiento de la sociedad con el propósito de incentivar un aprendizaje colectivo sobre estas obras, esto puede malinterpretarse para justificar la creación de otra obra que termine infringiendo el derecho de autor del primero, incurriendo así en plagio, acción contraria a derecho que supone en la imitación de la obra original. En busca de evitar dicha problemática, Hegel plantea el honor como un elemento esencial.

\subsection{Perspectiva contemporánea: Margaret Radin}

El desarrollo de la versión contemporánea de la teoría de la personalidad se atribuye en gran medida a los aportes de la profesora Margaret Radin, quien ha defendido la gran relevancia de la propiedad como elemento esencial en el desarrollo personal en su artículo llamado "Property and Personhood" (1982). En el texto, esta autora se centra en definir la teoría de la personalidad desde dos componentes básicos: la justificación de la existencia de los derechos de propiedad y su delimitación, demostrando un punto de vista en el que se crea y se construye el desarrollo personal en términos de "cosas" (Radin, 1982).

Un aspecto para resaltar es el uso de la expresión property rights, refiriéndose a los privilegios obtenidos del reconocimiento de la propiedad privada; este concepto se señala porque la profesora hace uso de dicha expresión en plural. Lo anterior puede tener dos signifi- 
cados: en primer lugar, el uso del plural puede entenderse desde la visión de que si bien la propiedad privada se entiende como una sola, al reconocerse se deriva la titularidad de varios derechos subjetivos. Pero, en segundo lugar, dicho uso de la expresión puede tener como objetivo definir el término de propiedad privada como un género del que emanan numerosas vertientes. Por motivos prácticos, el presente texto tomará como referencia el último significado en cuanto al desarrollo que la profesora Radin le da en su teoría.

Es necesario comprender el reto que significó traducir esta teoría del inglés al español, especialmente por la traducción de la expresión personhood perspective y personality theory, en cuanto a que ambas terminan siendo traducidas con la palabra "personalidad", lo cual no es adecuado con la terminología de estas expresiones, pues son distintas en el idioma inglés.

Respecto a la personhood perspective, Radin (1982) regresa al inicio de la teoría de la personalidad desde una perspectiva liberal, reafirmando la esencialidad de los conceptos de "voluntad" y "autodeterminación", elementos que también fueron desarrollados desde la perspectiva clásica de la teoría. No obstante, se resalta que si bien esta autora estudia a Kant y a Hegel, llega a una conclusión diferente, a la que nos referimos anteriormente como personhood perspective, argumentando que la personalidad surge en el momento en que lo personal, lo intangible, toma cuerpo o se construye a partir de objetos (Radin, 1982).

Al desarrollar la versión contemporánea planteada por Radin (1982), es necesario poner en conocimiento los seis pasos necesarios expuestos por la profesora, siendo el primero el desarrollo de la propiedad y de la personalidad desde un punto de vista intuitivo; en segundo lugar, expone las opciones que existen para abordar el concepto de "persona" con base en esto; el tercer punto es el desarrollo de un plan argumentativo; el cuarto paso muestra la justificación que le otorga a la protección de la propiedad desde un punto de vista moral y centrándose en la personalidad del individuo. Por otro lado, el quinto punto se encarga de determinar los retos que nacen en relación con figuras jurídicas; finalmente, demuestra la importancia de reconocer los derechos de propiedad desde la personalidad. Estos pasos se explican con más detalle a continuación: 
Perspectiva intuitiva de la propiedad y la personalidad: el punto clave de esta perspectiva es entender que la propiedad no se predica de igual manera sobre todos los objetos, debido a que existen otros a los que se les otorga un gran sentido de propiedad respecto a su propietario, fundamentado en un vínculo especial de valoración personal sobre ese objeto. Lo anterior, por la construcción que realiza el ser humano de su personalidad y que le atribuye a determinado objeto.

Dicho esto, Margaret Radin (1982) sostiene la relevancia de la autonomía, pues al afirmar que existe un vínculo constitutivo entre determinada persona y un objeto, se entiende que el sujeto tiene la libertad de ejercer dominio sobre este. En conclusión, la profesora resalta de esta perspectiva tres afirmaciones: la primera plantea la existencia de una importante relación entre la persona y los objetos que la rodean, llegando al punto en que afirma la imposibilidad del desarrollo de la personalidad sin antes crear este tipo de vínculo, entendiendo que dichos objetos hacen parte del entorno en el que se desenvuelve la persona; el segundo punto le encuentra fundamento al primero, pues afirma que dicho vínculo surge del ejercicio de la libertad del sujeto que lo une al objeto, partiendo desde el término de la personalidad, ya que esa relación trasciende el plano meramente material para pasar a la constitución de la personalidad a través del objeto; en tercer lugar, es necesario resaltar que esta perspectiva no logra dar una justificación completa respecto a la forma en que la personalidad otorga un reconocimiento o protección moral y jurídica sobre la propiedad.

Noción de "persona": dentro de esta teoría surge la necesidad de definir un criterio objetivo que tenga la capacidad de establecer qué es susceptible de protección y qué no lo es. Para esto, Radin (1982) propone definir el concepto de "persona" desde el estudio de las teorías libertarias, de tal manera que se pueda cumplir con lo anterior.

Para esta autora, a diferencia de lo planteado por Kant (1887), el vínculo que surge entre la persona y la propiedad no es un elemento esencial para el desarrollo de su teoría. En este argumento, la profesora parece encontrar un punto en común con Locke (1988), en cuanto a que este autor sostiene que el entorno que rodea a la persona necesariamente es parte de ella, debido a que la relación con los factores externos termina conformando la memoria del individuo. Sin embargo, Radin se separa de Locke, argumentando que 
este no incluyó taxativamente la premisa de que los objetos terminan siendo parte de la constitución de la persona. Lo anterior lleva a la profesora a desarrollar una nueva definición del concepto de "persona”, entendiéndola como una organización o estructura continua de carácter que integra los planes futuros, generando cierta expectativa con los sentimientos y eventos pasados, retomando lo expuesto por Bentham en cuanto a que "la idea de propiedad consiste en una expectativa establecida" (Radin, 1982).

Enfoque hegeliano: en este punto, Radin (1982) estudia la teoría hegeliana de la propiedad, otorgando varios argumentos novedosos dentro de este estudio. La profesora inicia señalando que tanto para Hegel como para Kant, el término "persona” hace referencia a la capacidad de ser titular de derechos, lo cual se queda atrás para su teoría, puesto que ella supone que el concepto solo se materializa en el momento en que, mediante la voluntad y la libertad, se genera una actuación en el entorno del individuo.

Aun así, las obras de Hegel influyen en gran medida a la teoría de la personalidad desarrollada por Radin (1982). La teoría hegeliana parte de la premisa de que el concepto de "propiedad" va ligado a la voluntad, generando el reconocimiento de un derecho sobre el objeto. En cuanto a esto, se debe entender que la voluntad debe materializarse en el objeto, pero que dicha materialización no puede ser reducida a una única vez, sino que debe contar con el elemento de la continuidad para poder reconocer el derecho a la propiedad sobre aquel objeto.

Finalmente, lo anterior puede resumirse en tres puntos clave que rescata Radin (1982) de la teoría hegeliana con el fin de aplicarlos a su personhood perspective: en primer lugar, resalta que el término "persona" va ligado con el entorno que lo rodea, pues su voluntad es materializada en objetos externos; esto significa que no puede existir por sí solo sin tener algún vínculo con su exterior. En segundo lugar, el concepto de "libertad" hegeliano debe ser interpretado a la luz de una libertad en grupo, mas no individual, en cuanto a que no solo implica la acción de dominio sobre el objeto, sino que también conlleva la capacidad de oponerse a interferencias de terceros. En último punto, sostiene la existencia de ciertos tipos de propiedad, los cuales conllevan una relación esencial con la personalidad. 
Protección de la propiedad desde la personalidad: en este paso se analizará la justificación que Radin (1982) le otorga a la propiedad en el desarrollo de su denominada personhood perspective. Plantea que dicha justificación sostiene la capacidad de ubicar a los derechos en un punto de continuidad entre lo personal y lo fungible. A simple vista, se podría afirmar que esa situación es contradictoria a ambas figuras de propiedad, pero la profesora no se queda en el simple planteamiento, sino que le da respuesta esclareciendo que al hablar de algo fungible, no siempre nos referimos a algo antagónico a lo personal. Dicha distinción surge debido a la naturaleza o gracias al vínculo entre los objetos con la personalidad, entendiendo que entre más cercano sea este vínculo, mayor protección de la propiedad se va a generar.

Continuando con el desarrollo del personhood perspective, Radin (1982) tiene como objetivo principal entender cuál es el propósito de un reconocimiento mayor de la propiedad sobre ciertos bienes, siendo la respuesta el elemento de la calidad o de la fuerza del vínculo entre la persona y el objeto, generando una categoría objetiva para entender el nivel de reconocimiento sobre los objetos. Con esto, la teoría contemporánea de la autora busca salir de un criterio meramente subjetivo para demostrar la necesidad de entender dichas relaciones subjetivas desde un plano objetivo.

Para dar fin a lo anterior, es necesario recapitular la importancia del alcance de la personhood perspective, puesto que incluyó en la teoría de la personalidad el novedoso planteamiento de que no todos los objetos tienen el mismo valor, ya que este depende de la fuerza del vínculo que se genere con una persona o de ser un objeto esencial para alcanzar un propósito establecido. Por otro lado, la personhood perspective tiene como fin plantear un criterio objetivo para entender las relaciones subjetivas entre sujeto-objeto, entendiendo que el ser persona va intrínsecamente ligado con los objetos encontrados en su entorno sobre los que ejerce su voluntad, centrándose en establecer si el objeto puede ser constitutivo de la personalidad en vez de en el interés personal que se busca alcanzar con dicho objeto (Radin, 1982). Finalmente, Radin (1982) se pronuncia acerca del derecho de autor en específico dando un breve bosquejo al argumentar que, en este caso, la propiedad de objetos comerciales no deriva necesa- 
riamente de la personalidad de su dueño, a diferencia de lo que pasa con un discurso público.

\section{La teoría de la personalidad y la creación de obras por medio de la IA}

Desde la versión kantiana de la teoría de la personalidad, la IA no podría ser considerada como titular de ningún tipo de derechos sobre las obras que crea. El ejercicio de la libertad, para Kant (1887), se entiende desde un plano objetivo y un plano subjetivo. El primero hace referencia a la posesión sensible del bien, algo que difícilmente podría lograrse si hablamos de sistemas que se ubican en un plano completamente distinto al exclusivamente físico o tangible. Además, frente al plano subjetivo del ejercicio de la libertad, debería tratarse de sistemas que pudieran dar muestras de una posesión racional, es decir, de experimentar algún tipo de sentido de pertenencia por un vínculo con la obra. En otras palabras, la IA puede dar lugar a obras, pero sobre ellas la falta de conciencia sobre un vínculo directo con un "ego trascendental" (aunque sea este un término de Hegel) imposibilita que podamos hablar de una propiedad derivada del ejercicio de la libertad, entendida como posibilidad de autodeterminación frente a fines existenciales.

$\mathrm{Si}$ analizamos lo anterior a partir de los planteamientos hegelianos, la noción misma de IA supone la imposibilidad de pensar en algún tipo de propiedad. Teniendo en cuenta que para este filósofo la propiedad proviene de la encarnación de un yo abstracto a través del contenido que se le da desde el mundo exterior, la IA (asumiendo incluso que podría tener autoconciencia) se encuentra determinada por naturaleza. Si el proceso creativo detrás de las obras creadas por IA responde a un algoritmo predeterminado y diseñado por otro que elimina la posibilidad de un desarrollo personal libre, tampoco existiría propiedad alguna. No es este el caso del ser humano, incluso para aquellos que somos creyentes; si bien contamos con una naturaleza de ser criaturas, podemos cultivar los gustos a nuestro antojo. En el caso de la IA, el diseñador del programa que actúa desde la posición que podría pensarse de forma análoga a la de Dios no lo hace de la misma manera, desde el diseño mismo le impone a la IA 
la creación de una obra a partir de datos suministrados y la información que es extraída por el sistema.

En el caso de la versión de Radin (1982) de la teoría de la personalidad, el panorama es aún más limitado para los defensores de un reconocimiento tradicional de derechos para las obras creadas por IA. Si lo que define una teoría de la propiedad basada en la personalidad, además del ejercicio de la libertad, es la noción misma de persona ligada a la memoria, el presente y las expectativas futuras, la IA está lejos de ser considerada como persona y aún más distante de poder dar lugar a algún tipo de derecho de propiedad. En este sentido, si realmente existen bienes que cuentan con un reconocimiento de la propiedad diferenciado por su vínculo con el dueño, en la medida en que no podemos hablar de una persona humana libre y autónoma frente a su proyecto de vida, no podríamos hablar de algún tipo de reconocimiento de derechos.

Sin embargo, como se mostró en el texto, la filosofía del derecho de autor suele ser flexible con el fin de dar respuesta a los retos que van surgiendo en nuestras sociedades. $\mathrm{Y}$ es que, en este punto del análisis, resulta un poco confuso llegar a una conclusión sin lugar a duda sobre la protección o no de dichas obras. Para poder dilucidar esto, es necesario remitirnos al elemento de la impredecibilidad que se encuentra en dichas tecnologías -elemento que puede verse como la originalidad de las obras creadas por estos sistemas- y al estudio realizado de las relaciones que surgen entre el autor, la creación y la sociedad, conectando la teoría de la personalidad con la teoría cultural y la importancia de construir un acervo común con el conocimiento adquirido de las nuevas creaciones.

\section{Conclusiones}

De lo anterior concluimos que si bien no se puede afirmar que la IA es susceptible de recibir el reconocimiento de protección desde una visión tradicional, resulta de especial relevancia el aseguramiento del conocimiento y la construcción de canales de información que surgen producto de este tipo de desarrollos tecnológicos. Así, resulta necesario que el sistema de derecho de autor otorgue algún tipo de protección a las creaciones desarrolladas por la IA. Una propuesta 
para ello se encuentra en La teoría de la personalidad y la inteligencia artificial: una respuesta desde lo clásico con la mirada contemporánea del acervo común (Páez, 2021), en el cual se asume un compromiso con tres factores fundamentales: i) el reconocimiento de este tipo de obras creadas por IA y la posibilidad de su protección como una necesidad práctica para salvaguardar el desarrollo, la inversión y la asunción de esfuerzos detrás de los procesos de investigación y desarrollo en pos de la sociedad; ii) la carencia de un conocimiento común sobre el alcance y las implicaciones de la IA; y iii) la insuficiencia de las posturas adoptadas en la actualidad en la mayoría de países del mundo respecto a la IA.

De lo anterior se desprende que el sistema de derecho de autor, como cualquier otro sistema normativo, debe ser capaz de adaptarse a las necesidades prácticas que surgen con el paso del tiempo. Usualmente, la respuesta a este tipo de planteamientos es la aparición de nuevas fuentes normativas que busquen aclarar cuál es la forma correcta de aplicación de la ley frente a la nueva necesidad. Sin embargo, la propuesta que se intentó exponer en este escrito fue diferente, en el sentido de que procuró una exposición de elementos esenciales de los sistemas de derecho de autor, un regreso a sus aspectos fundamentales para que el espíritu de los sistemas normativos de derecho de autor pudiera ofrecer una respuesta que no altere las figuras jurídicas existentes, dada la extrema complejidad que eso supondría.

Por estas razones, si es necesario formular una propuesta frente a la protección o no protección de obras creadas por medio de la IA, lo cierto es que dicha alternativa debe ser respetuosa de los esfuerzos y recursos invertidos para que este tipo de obras sean creadas, la apertura a nuevos canales y fuentes de información valiosa para la construcción de un acervo común que se nutra tanto de lo clásico como de las formas contemporáneas de producción, y respetuosa del derecho de autor mismo. Es por esto último que la búsqueda de la respuesta frente a la protección de obras protegidas por IA se inició desde una de las teorías más cercanas al sistema de derecho de autor contemporáneo, la teoría de la personalidad, que, como se expuso anteriormente, logra poner en evidencia la necesidad de contar con sistemas protectores de ese vínculo inmaterial del autor y la obra. Al intentar establecer un paralelo con la IA, resulta difícil aplicar esta 
teoría de forma análoga a como se aplica sobre la creación de obras del ser humano, por eso es preciso incluir elementos adicionales que refuercen la justificación de la protección de este tipo de obras para no desatender esos factores determinantes relacionados con la necesidad práctica que supone encontrar una respuesta que, a su vez, no desincentive el interés y la inversión en este tipo de desarrollos tecnológicos.

Así, encontramos que si bien una aplicación rígida de la teoría de la personalidad no es posible, lo cierto es que el alcance de la personalidad, como se expuso en el apartado cuatro, también tiene un componente esencial de protección del ejercicio de la libertad que solo es posible desde la relación de los autores con el medio exterior y la cultura que los rodea. La construcción de la propia personalidad ${ }^{9}$ es posible, en gran medida, gracias a la existencia de un acervo desde el cual es posible hablar en términos de desarrollo. Así como en los sistemas de patentes, al hablar de la novedad como requisito de patentabilidad, se hace referencia al estado de la técnica para conocer dónde se ubica el objeto de protección y en qué consiste el desarrollo que ofrece, en términos culturales, el conjunto de saberes, prácticas, conocimientos e información representa ese punto de partida del que se nutre la producción literaria, artística y científica.

Ese conjunto al que se hizo referencia es el lugar a donde llegan las obras protegidas por medio de IA. De allí que la aplicación de la teoría de la personalidad, si bien no se puede dar de forma rígida por la incongruencia que representa buscar en un sistema artificial elementos subjetivos que trascienden el mero relacionamiento de datos, sí podemos buscar una respuesta desde una teoría de la personalidad por sus implicaciones sobre la sociedad y no fundamentada de manera exclusiva en el sujeto. Esto podría representar de alguna manera que se estuviera buscando forzosamente la aplicación de la mencionada teoría a la problemática que supone la creación de obras por medio de IA, pero no debemos olvidar que es este un problema del hoy que necesita respuesta y la teoría de la personalidad cuenta con elementos que nos pueden acercar a ella.

9 Como puede encontrarse en las obras de Amartya Sen y de Martha Nussbaum al estudiar la figura de la vida plena. 
La protección de la personalidad de un autor y su vínculo con las obras que crea parten del ejercicio de su libertad y de la capacidad creativa que plasma en su trabajo. Sin embargo, esa personalidad ligada de forma esencial al producto final, e incluso la obra en sí misma, no son creaciones ex nibilo, provienen de esa subjetividad y esfera personal que el autor ha forjado desde su contacto y proceso nutritivo desde el acervo común. Si la IA supone que ese acervo común continúe engrandeciéndose, el derecho de autor no puede ser un obstáculo para ello. El sinsentido que supone pensar en que se reconozca autoría en un sistema artificial ${ }^{10}$ debe convertirse en una pregunta para encontrar la forma más idónea y justa de incentivar - como vimos que proponía la teoría utilitarista- la producción a partir de procesos creativos.

Teniendo en cuenta lo anterior, desde la teoría de la personalidad es posible pensar que es necesaria la protección de las obras protegidas por medio de la IA como una forma de velar por el ejercicio de la libertad y la autonomía de los autores, sin que ellos ni la sociedad vean limitado su acceso al conocimiento. Esa protección se traduce en la salvaguarda del acervo común como un elemento propio de la teoría cultural, pero que nos es útil para darle nombre a la necesidad que supone el no desatender la inversión de recursos de toda índole detrás de la IA. De allí que nuestra propuesta, como pudo intuirse a lo largo del texto y como parece aceptarse actualmente, consiste en el reconocimiento de derechos en cabeza de aquel que asume los esfuerzos necesarios para que la IA exista.

Así como en el caso de las presunciones de transferencia de derechos de las obras por encargo a favor del encargante, o de la presunción de titularidad en cabeza de los productores de obras audiovisuales -por ser aquellos "por cuya cuenta y riesgo" es posible la obra-, la IA podría recibir un tratamiento análogo en el que exista una debida protección de los intereses de los que se ha venido hablando, así como un debido respeto a las figuras jurídicas que componen los sistemas de derecho de autor. Pese a que parece una solución fácil y podría existir quien la tildara de evidente, lo cierto es que hay un

10 Ya que muchos de nuestros sistemas actuales establecen que la autoría debe ser necesariamente humana. 
sustento filosófico para demostrar su viabilidad; a esta justificación es a la que se espera que el lector haya podido llegar con la lectura de este texto. Después de todo, si algo nos ha demostrado la tecnología es que pulsar un botón artificial en una pantalla es, en igual medida, tan sencillo como compleja es la estructura y el funcionamiento que lleva detrás, en ese terreno desconocido para el usuario, pero que se esconde siempre detrás de las imágenes que ve. Que sea este el caso para que la justificación y el fundamento muchas veces ocultos y poco abordados por la preocupación práctica del momento reciban al menos un leve haz de luz que nos lleve a indagar por respuestas sólidas que tengan una base desde la que podamos responder, en último término, de una forma justa.

\section{Bibliografía}

Antequera Parilli, R. (2007). Estudio de Derecho de Autor y Derechos Afines. Reus. Arendt, H. (2015). La condición Humana. Vida activa y condición humana (2a ed.). Paidós.

Attas, D. (2008). Lockean Justification of Intellectual Property. En Gosseries, A. Marciano, A. y Strowel, A., Intellectual Property and Theories of Justice. Palgrave Macmillan.

Bécourt, D. (1990). La Revolución Francesa y el Derecho de Autor: Por un Nuevo Universalismo. Boletín de Derecho de Autor, XXIV(4).

Boden, M. (1998). Computer Models of Creativity. En Sternberg, R., Handbook of Creativity (pp. 351-372). Cambridge University Press.

Bringsjord, S. y Govindarajulu, N. S. (2018). Artificial Intelligence. Stanford Encyclopedia of Philosophy.

Carbonell, C. (2015). Platón visita una exposición de Malévich. El arte en la ampliación de la experiencia filosófica. En Llano, A., El arte más allá de sí mismo. Aproximaciones a la cultura artística contemporánea (pp. 91-109). Siglo XXI.

Chiassoni, P. (2011). Técnicas de Interpretación Jurídica. Marcial Pons.

Cohen, J. (2007). Creativity and Culture in Copyright Theory. UC Davis Law Review, 40, 1151-1205.

Comisión de la Unión Europea. (2018). Communication from the Commission to the European Parliament, the European Council, the Council, the European Economic and Social Committee and the Committee of the Regions. Artificial Intelligence for Europe SWD(2018) 137 final.

Córdoba, J. F. (2015). El Derecho de Autor y sus Limites (1ª ed.). Temis. 
Dock, M. C. (1974). Génesis y evolución de la noción de propiedad literaria. Revue Internationale du Droit D'Auteur (RIDA).

Drahos, P. (2016). A Philosophy of Intellectual Property. Australian National University eText.

Draper, J. W. (1921). Queen Anne's Act: A Note on English Copyright. Modern Language Notes, 36(3), 146-154.

Du Bois, M. (2018). Justificatory Theories for Intellectual Property Viewed through the Constitutional Prism. University of South Africa: PER/PELJ.

Durante, M. (2019). Potere computazionale. L'impatto delle ICT su diritto, società, sapere (Italian edition). Meltemi.

Dworkin, R. (1978). Liberalism. En Hampshire, S., Public and Private Morality (pp. 113- 143). Cambridge University Press.

Espejo, J. C. (2020). ¿Cuáles son las fronteras en los Procesos de Desarrollo de la IA? En Inteligencia Artificial. Transformaciones y retos en el sector editorial (pp. 18-32). CERLALC/UNESCO.

Fariñas, J. R. (2020). Inteligencia Artificial y derecho de autor: consideraciones sobre la autoría y la titularidad. En Inteligencia Artificial. Transformaciones y retos en el sector editorial (pp. 33-50). CERLALC/UNESCO.

Fisher, W. (2001). Theories of Intellectual Property. En New Essays in the Legal and Political Theory of Property (pp. 168-199). Cambridge University Press.

Geller, P. E. (1994). Must Copyright be Forever Caught between Marketplace and Authorship Norms? En Sherman, B. y Strowel, A., Of authors and origins. Oxford University Press.

Gervais, D. J. (2008). Intellectual Property and Human Rights: Learning to Live Together. Kluwer.

Gervais, D. J. (2019). The Machine As Author. Iowa Law Review, 105, 2053-2106.

Ginsburg, J. (1997). Author and Users in Copyright. Journal of the Copyright Society of the U.S.A., 45(1), 1-20.

Ginsburg, J. (2019). Informe General, Justificación del los Derechos de Autor. En Blomqvist, J., Copyright to Be or not to Be. ALAI. Ex Tuto.

Ginsburg, J. C. y Budiardjo, L. A. (2019). Authors and Machines. Berkeley Technology Law Journal, 3, 343-456.

Gordon, W. (1993). A Property Right in Self-Expression: Equality and Individualism in the Natural Law of Intellectual Property. Yale Law Journal, 102, 1533-1609.

Hamet, P. y Trembaly, J. (2017). Artificial Intelligence in Medicine. Metabolism. Hegel, G. (1966). Fenomenología del Espiritu. Fondo de Cultura Económica.

Hegel, G. (1991). Elements of the Philosophy of Right. Cambridge University Press. Hervada, J. (2002). ¿Qué es el Derecho? Eunsa.

Hettinger, E. (1989). Justifying Intellectual Property. Philosophy and Public Affairs, 18(1), 31-52.

Holzinger, A., Langs, G., Denk, H., Zatioukal, K. y Müller, H. (2019). Causability and Explainability of Artificial Intelligence in Medicine. WIREs. 
Huges, J. (1998). The Philosophy of Intellectual Property. Georgetown University. Kant, I. (1887). The Philosophy of Law. T. \& T. Clark.

Kant, I. (1973). Critica del juicio. Porrúa.

Kateb, G. (1992). The inner Ocean: Individualism and Democratic Culture. Cornell University Press.

Landes, W. M. y Posner, R. A. (2003). The Economic Structure of Intellectual Property Law. Harvard University Press.

Lipszyc, D. (2017). Derecho de Autor y Derechos Conexos. CERLALC.

Litman, J. (1990). The Public domain. Emory Law Journal, (39), 965-1023.

Locke, J. (1988). Two Treatises of Government. Cambridge University Press.

Menell, P. (2000). Intellectual Property: General Theories. En Bouckaert, B. y De Geest, G., Encyclopedia of Law \& Economics (Vol. II, pp. 129-188). Cheltenham.

Merges, R. P. (2011). Justifiying Intellectual Property. Harvard University Press .

Mills, M. (23 de febrero de 2016). Artificial Intelligence in Law: The State of Play 2016. Thomson Reuters Legal Executive Institute blog. https://www.legalexecutiveinstitute.com/artificial-intelligence-in-law-the-state-of-play-2016part-1/.

Moore, A. (2015). Lockean Foundations of Intellectual Property. The WIPO Journal - Analysis of Intellectual Property Issue, 7(1), 29-40.

Munzer, S. R. (1993). Kant and Property Rights in Body Parts. Canadian Journal of Law and Jurisprudence, 6, 319-342.

Netanel, N. (1992). Copyright alienability restrictions and the enhancement of author autonomy: normative evaluation. Rutgers Law Journal, 24(2), 347-442.

Nozick, R. (1974). Anarchy, State, and Utopia. Basic Books.

Nussbaum, M. (2000). Women and Human-Development: The Capabilities Approach. Cambridge University Press.

Nussbaum, M. (2003). Capabilities as Fundamental Entitlements. Feminist Economics, 9(2-3), 33-59.

OCDE. (s.f.). Recommendation of the Council on Artificial Intelligence. OECD/ LEGAL/0449.

Páez, P. (2021). La teoría de la personalidad y la inteligencia artificial: una respuesta desde lo clásico con la mirada contemporánea del acervo común. Universidad de La Sabana.

Poole, D., Mackworth, A. y Goebel, R. (1998). Computational Intelligence. A logical approach. Oxford University Press.

Radin, M. J. (1982). Property and Personhood. Stanford Law Review, 34(5), 9571015.

Robinson, D. L. (1991). On the Neurology of Intelligence and Intelligence Factors. En Rowe, H., Intelligence Reconceptualization and measurement. Australian Council for Educational Research.

Sancho Caparrini, F. (2018). Breve Historia de la Inteligencia Artificial. Revista de Occidente, (446-447), 19-33.

Schwab, K. (2016). La Cuarta Revolución Industrial. Debate. 
Shiffrin, S. (2001). Lockean Arguments for Private Intellectual Property. En New Essays in the Legal and Political Theory of Property. Camridge Studies in Philosophy and Law.

Steiner, C. (1998). Intellectual Property and the Right to Culture. Panel Discussion on Intellectual Property and Human Rights. World Intellectual Property Organization. https://www.wipo.int/edocs/mdocs/tk/en/wipo_unhchr_ip_pnl_98/wipo_unhchr_ip_pnl_98_2.pdf.

Stengel, D. (2004). La propiedad intelectual en la filosofía. Revista La Propiedad Inmaterial, 8, 71-106.

Sternberg, R. J. (2013). Intelligence. En Freedheim, D. K. y Weiner, I. B., Handbook of psychology: History of psychology (pp. 155-176)). John Wiley \& Sons, Inc.

Tatarkiewicz, W. (1992). Historia de seis ideas. Arte, belleza, forma, creatividad, mímesis, experiencia estética (Presentación B. Dziemidok, Trad. F Rodríguez Martín). Tecnos.

Treiger-Bar-Am, K. (2008). Kant on Copyright: Rights on Transformative Authorship. Cardozo Arts \& Entertainment Law Journal, 25(3), 1059-1104.

Treiger-Bar-Am, K. (2016). Copyright and Positive Freedom: Kantian and Jewish Thought on Authorial Rights and Duties. Journal of the Copyright Society of the USA, 63(4), 551-572.

Velázquez, L. (2004). Bienes (9a ed.). Temis.

Woodmanse, M. (1984). The Genius and the Copyright: Economic and Legal Conditions of the Emergence of the 'Author'. Eighteenth-Century Studies, 17(4). 425-448.

Yoo, C. S. (2019). Rethinking Copyright and Personhood. Faculty Scholarship at Penn Law, (423), 1039-1077. 